\title{
Galectin Lattice Regulates Nutrition Sensor Functions in Pancreatic $\beta$ Cells
}

\author{
Kento Maeda $^{1}$; and Kazuaki Ohtsubo ${ }^{1,2}$ \\ ${ }^{1}$ Department of Analytical Biochemistry, Graduate School of Health Sciences, Kumamoto University, Kumamoto 862-0976, Japan \\ ${ }^{2}$ Department of Analytical Biochemistry, Faculty of Life Sciences, Kumamoto University, Kumamoto 862-0976, Japan \\ FAX: +81-96-373-5467, TEL: +81-96-373-5467, E-mail: kohtsubo@kumamoto-u.ac.jp
}

(Received on June 29, 2018, accepted on August 7, 2018)

Key Words: glucose transporter, galectin lattice, cationic amino acid transporter, pancreatic $\beta$ cell

\begin{abstract}
Recently, it has been elucidated that the lattice structure formed by complexes of galectins and $\mathrm{N}$-glycans regulates expression and function of plasmalemmal glycoproteins, which is important for maintaining various homeostatic systems. We have previously revealed that the failure of $\mathrm{N}$-glycosylation abolishes lattice formation and induces aberrant membrane sub-domain distribution of Glucose transporter 2 (GLUT2) in pancreatic $\beta$ cells that consequently attenuates the cellular glucose uptake function. This results in the impairment of insulin secretion in pancreatic $\beta$ cells in the disease process of diabetes. Although the biological significance of the formation of the galectin lattice is well recognized, the molecular regulatory mechanism of the localization and function of plasmalemmal glycoproteins has not been clarified. To address the question, we analyzed the component proteins of the galectin lattice complexes on pancreatic $\beta$ cell surface and found that the lattice complexes were composed of Cationic amino acid transporter 3 (CAT3), Teneurin-3, Myosin-4, Actin, $\alpha$-Tubulin, and GLUT2 at least. These results indicated that the galectin lattice forms clusters of plasmalemmal glycoproteins through galectin- $N$-glycan bindings and further suggested the presence of the common mechanism among the functional regulation of nutrition sensors in the insulin secretion of pancreatic $\beta$ cells.
\end{abstract}

\section{A. Introduction}

Mammalian cells produce a highly complex structural repertoire of $N$-glycans, which present a variety of glycan epitopes on multiple glycan branches. These glycan moieties are recognized by diverse endogenous proteins, so called lectins. The binding affinity of lectins to glycoproteins is determined by the number of corresponding glycan epitopes intrinsically assigned by the amino acid sequence motifs of glycoproteins for $N$-glycosylation and the antennal complexity of the carried individual $N$-glycans. The architectural representation of glycan moieties depends on the activity of glycan synthesis pathway, which is reflecting cellular physiological microenvironments through the genetic regulation of glycosyltransferase expression and metabolic regulation for the production of nucleotide sugars (1).

We have previously revealed that intake of high-fat diet elevates oxidative stress in pancreatic $\beta$ cells that subsequently diminishes the expression of GnT-IVa glycosyltransferase via the transcriptional attenuation by induction of the nuclear exclusion of responsible transcription factors. This, thereby, impairs the branch formation of complex type $N$-glycans $(2,3)$. Even though the high fat diet-induced dysglycosylation was detected on various glycoproteins in pancreatic $\beta$ cells, the effect was obvious especially on the in situ distribution and function of Glucose transporter 2 (GLUT2). It has been reported that high fat diet administration induces mislocalization of GLUT2 and impairs the glucose stimulated insulin secretion of pancreatic $\beta$ cells associating with type
2 diabetes in rodents (4). Cell surface residency of GLUT2 was significantly reduced and it intracellularly accumulated especially in early endosomes and lysosomes (2). The mislocalizaion of GLUT2, therefore, impairs cellular glucose uptake function and abolishes glucose stimulated insulin secretion of pancreatic $\beta$ cells in high-fat diet administrated mice. Moreover, the genetic elimination of GnT-IVa in mice caused the misglycosylation of GLUT2 and subsequently induced mislocalization of that in pancreatic $\beta$ cells. The GnT-IVa deficient mice exhibited impaired glucose stimulated insulin secretion of $\beta$ cells and symptoms diagnosed as type 2 diabetes (2). These findings provided clues indicating the presence of the mechanism regulating membrane localization of GLUT2 related to glycan recognition.

\section{B. GLUT2 Expression and Galectin}

As primary isolated pancreatic $\beta$ cells from GnT-IVa deficient mice showed the reduced cell surface residency and the intracellular accumulation of GLUT2, GLUT2 might bind to plasmalemmal anchoring molecule recognizing GLUT2 $N$-glycan branch structures. Structural analyses revealed that GLUT2 $N$-glycans had multiantennary branch structures bearing lactosamine moieties. Consistent with the speculation, addition of synthetic glycans mimicking GLUT2 glycan branches to the culture of primary isolated pancreatic $\beta$ cells reduced cell surface expression levels of GLUT2. Since $\beta$-galactosides showed significant reductions of cell surface residency of GLUT2, galectins were predicted as the 
candidate of the anchoring molecule. Analyses of GLUT2 protein complex conjunction with cell surface protein cross-linking revealed that Galectin-9 substantially bound to GLUT2 that was inhibited by treatment with $\beta$-galactosides in dose-dependent manner (2). These results indicated that the GnT-IVa dependent GLUT2 glycosylation provides ligands binding to Galectin-9 and stabilizes GLUT2 on cell surface through the formation of galectin-glycan complexes, so called lattice.

\section{Membrane Sub-Domain Distribution of GLUT2}

Plasmalemmal proteins reside in various membrane sub-domains and form clusters through homotypic and heterotypic interactions. The protein composition is dynamically exchanged among those membrane sub-domains in response to extrinsic and intrinsic cellular signals. Although the biological significance of the lattice formation in the stabilization of GLUT2 protein on $\beta$ cell surface was well understood, the regulatory mechanism of the membrane sub-domain distribution of GLUT2 has been remained unclear. It has been previously reported that the glucose transport activity of GLUT1, which is a member of GLUT family, is controlled by its re-distribution among membrane sub-domains $(5,6)$. Lipid raft microdomain has Stomatin, which inhibits GLUT1 activity; thereby the re-distribution of GLUT1 into the lipid raft microdomain suppresses the cellular glucose uptake function. The high sequence similarity between GLUT1 and GLUT2 intimated the presence of the common mechanism regulating the function of GLUTs. We have revealed that GLUT2 localizes in non-lipid raft microdomains in normal pancreatic $\beta$ cells, though the disruption of the glycangalectin lattice by the treatment with synthetic glycans or genetic elimination of GnT-IVa induced the re-distribution of GLUT2 into lipid microdomains. The distribution shift allowed GLUT2 bound to Stomatin that inhibited its glucose transport activity (7). The dysglycosylation induced aberrant membrane distribution of GLUT2 is presumably involved in the deficit of $\beta$ cell function in the pathogenesis of type 2 diabetes.

\section{Exploring GLUT2 Anchoring System}

These findings suggested the presence of the mechanism anchoring GLUT2 protein in non-lipid raft membrane microdomains, in which the glycan-galectin lattice selectively gathers glycopro-

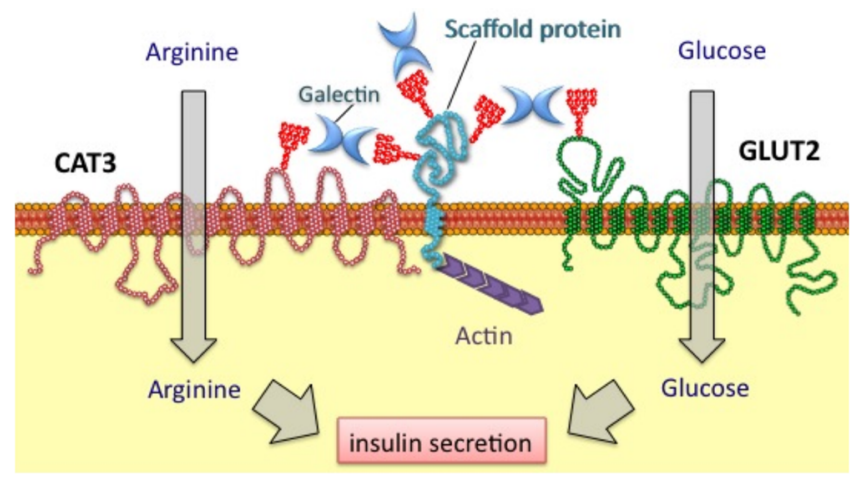

Fig. 1. Glycan-dependent formation of nutrition sensor clusters in pancreatic $\boldsymbol{\beta}$ cells. $\quad N$-glycan-galectin lattice enable to form a cluster of nutrition sensors consisting of GLUT2 and CAT3 by inter cross-linking with a scaffold protein. This facilitates the passive transport of nutrient molecules and stimulates the insulin secretion signal.

teins in the extracellular space and associates with cytoskeletal system in cytoplasm. To address this issue, we analyzed composition of the glycan-galectin lattice complexes in $\beta$ cells by mass spectrometry. We found that the galectin lattice is composed of Cationic amino acid transporter 3 (CAT3), Teneurin-3, Myosin-4, Actin, $\alpha$-Tubulin, and GLUT2 at least. Consistent with our speculation, we could detect various cytoskeletal proteins in the lattice complexes. Teneurin family proteins exhibit highly conserved structural similarities and have a large number of $N$-glycans presenting galectin ligands. Since Teneurin-1 has a domain to interact with a cytoskeleton adaptor protein, Tenrurin-3 presumably has a potential to bind to cytoskeletal proteins and works as a scaffold for extracellular glycoproteins and intracellular cytoskeletons (8). Pancreatic $\beta$ cells possess sensor molecules detecting physiological metabolic states for the appropriate insulin secretion regulating the biological processes coordinately maintaining energy homeostasis. CAT3 is one of the nutrition sensor molecules of pancreatic $\beta$ cells and is responsible for the amino acid-stimulated insulin secretion. As well as GLUT2, CAT3 belongs to the group of the solute carrier membrane transporters and bears multiple membrane spanning regions, and a single $N$-glycan in the extracellular loop. Curiously, these results suggest that functions of nutrition sensors can be regulated by common mechanism in pancreatic $\beta$ cells, in which sensor molecules form functional clusters in membrane microdomains by glycan-galectin lattice formation (Fig. 1)

\section{References}

1. Taniguchi, N. (2009) J. Biol. Chem. 284, 34469-34478.

2. Ohtsubo, K., Takamatsu, S., Minowa, M. T., Yoshida, A., Takeuchi, M., and Marth, J. D. (2005) Cell 123, 1307-1321.

3. Ohtsubo, K., Chen, M. Z., Olefsky, J. M., and Marth, J. D. (2011) Nat. Med. 17, 1067-1075.

4. Reimer, M. K., and Ahrén, B. (2002) Diabetes 51(Suppl. 1), S138-S143.

5. Barnes, K., Ingram, J. C., Bennett, M. D. M., Stewart, G. W., and Baldwin, S. A. (2004) Biochem. J. 378, 343-351.

6. Kumar, A., Xiao, Y.-P., Laipis, P.-J., Fletcher, B. S., and Frost, S. C. (2004) Am. J. Physiol. Endocrinol. Metab. 286, E568-E576. 
7. Ohtsubo, K., Takamatsu, S., Gao, C., Korekane, H., Kurosawa, M. K., and Taniguchi, N. (2013) Biochem. Biophys. Res. Commun. 434, 346-351.

8. Young, T. R., and Leamey, C. A. (2009) Int. J. Biochem. Cell Biol. 41, 990-993.

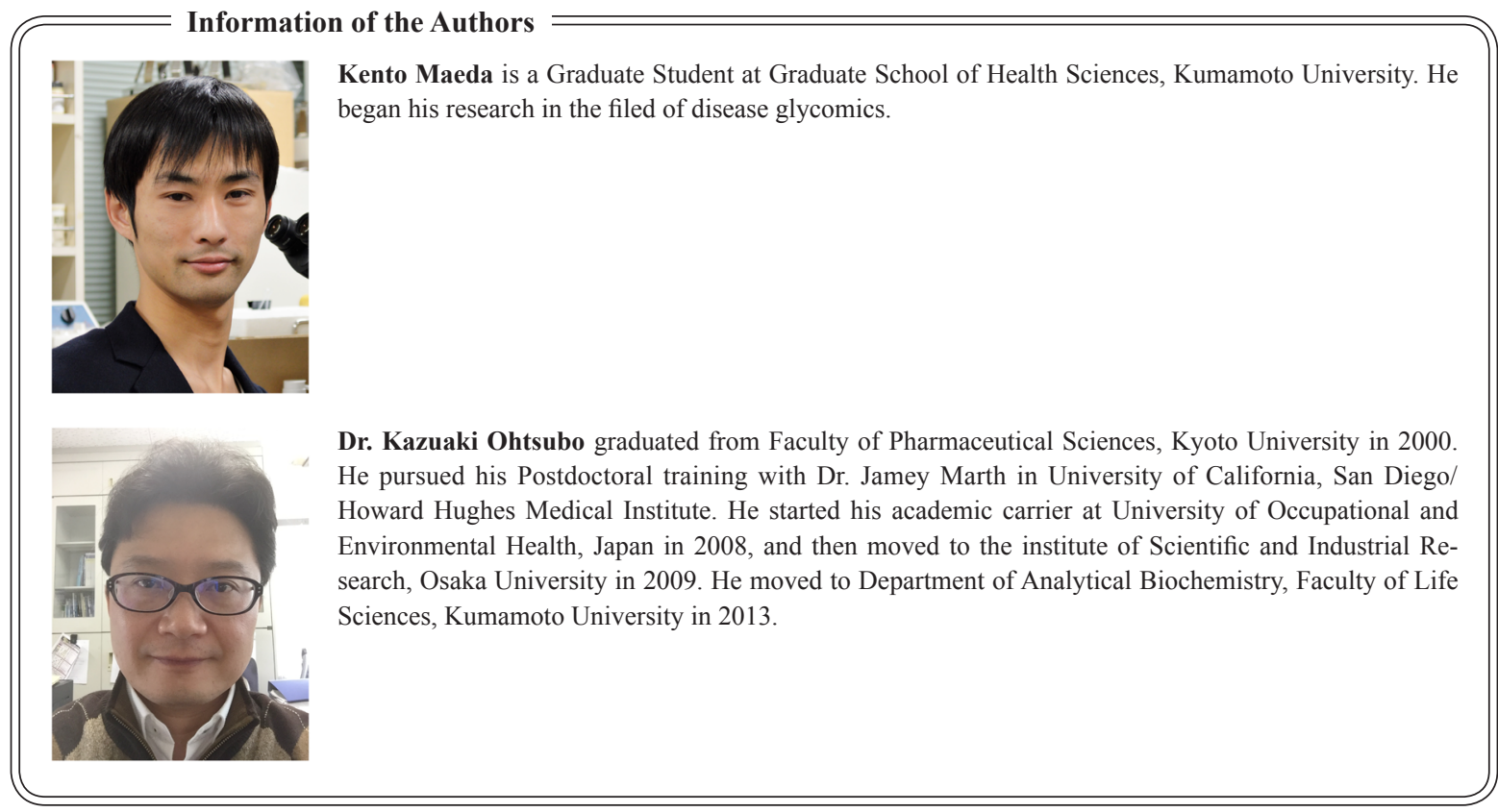

